Georgian Mathematical Journal

Volume 14 (2007), Number 3, 435-444

\title{
ON NONMEASURABLE SUBGROUPS OF UNCOUNTABLE SOLVABLE GROUPS
}

\author{
ALEXANDER KHARAZISHVILI AND ALEKS KIRTADZE \\ Dedicated to the memory of Academician I. Vekua \\ on the occasion of his 100th birth anniversary
}

\begin{abstract}
It is shown that any uncountable commutative group $(G,+)$ admits a representation $G=\cup\left\{G_{j}: j \in J\right\}$, where $J$ is a countable set, $G_{j}$ is a subgroup of $G$ for each $j \in J$, and $G / G_{j}$ is uncountable. A related fact for uncountable solvable groups is established in terms of invariant (quasiinvariant) measures and an application of the above-mentioned results to the measure extension problem is given.
\end{abstract}

2000 Mathematics Subject Classification: 28A05, 28D05.

Key words and phrases: Commutative group, solvable group, quasiinvariant measure, invariant measure, nonmeasurable subgroup, extension of measure, nonseparable measure.

It is well known that the basic ideas and concepts of the modern theory of dynamical systems came from the classical theory of ordinary differential equations. Indeed, for a given autonomous system of first-order ordinary differential equations in a finite-dimensional phase space, the famous Liouville theorem states the necessary and sufficient conditions under which the corresponding phase flow preserves the Lebesgue measure. These conditions are trivially fulfilled for Hamiltonian system in the same phase space. Consequently, we obtain a natural example of a dynamical system which is systematically used in studying various physical (primarily, evolutional) processes.

An abstract dynamical system can be treated as a triplet $(E, G, \mu)$, where $E$ is a nonempty phase space, $G$ is some group of transformations of $E$ and $\mu$ is a nonzero finite (or $\sigma$-finite) $G$-invariant measure on $E$.

Many deep and important results were established in terms of $(E, G, \mu)$. Among them there are: Poincare's recurrence theorem, Birkhoff's ergodic theorem, Krylov-Bogoliubov theorem on the existence of a dynamical system for a one-parameter group $G$ of homeomorphisms of a nonempty compact space $E$. The latter result was essentially generalized by Markov and Kakutani to a solvable group $G$ of homeomorphisms of a nonempty compact space $E$. This generalization shows, in particular, the remarkable role of solvable transformation groups in the theory of dynamical systems (equivalently, in the theory of invariant measures).

The main goal of the present paper is to discuss the measure extension problem for uncountable solvable groups. Namely, it will be demonstrated below 
that special properties of these groups enable one to extend a given invariant measure (i.e., a dynamical system) by using some nonmeasurable subgroup.

First, we would like to recall Vitali's classical construction of a Lebesgue nonmeasurable set on the real line. Let $\mathbf{R}$ denote the real line and let $\lambda$ be the Lebesgue measure on $\mathbf{R}$. Consider the Vitali partition of $\mathbf{R}$ determined by the equivalence relation $V(x, y)$ :

$$
(x, y) \in V \Leftrightarrow x \in \mathbf{R} \& y \in \mathbf{R} \& x-y \in \mathbf{Q},
$$

where $\mathbf{Q}$ denotes the set of all rational numbers. It is well known that any selector $Z$ of this partition is nonmeasurable with respect to $\lambda$ (see, e.g., [16]). Moreover, it can be shown that $Z$ is nonmeasurable with respect to any measure on $\mathbf{R}$ extending $\lambda$ and invariant with respect to the group of all rational translations of $\mathbf{R}$ (cf. [16], [17]). Among the selectors of the Vitali partition (which are usually called Vitali sets) we can encounter some subgroups of the additive group $\mathbf{R}$. Indeed, if we treat $\mathbf{R}$ as a vector space over the field $\mathbf{Q}$, then we can apply a well-known theorem from linear algebra, which states that a vector subspace $\mathbf{Q}$ of $\mathbf{R}$ admits a complemented subspace in $\mathbf{R}$, i.e., we have a representation

$$
\mathbf{R}=\mathbf{Q}+H(\mathbf{Q} \cap H=\{0\}),
$$

where $H$ is a vector space over $\mathbf{Q}$ (actually, $H$ is a hyperplane in $\mathbf{R}$ complemented to the line $\mathbf{Q}$ in $\mathbf{R}$ ). Consequently, $H$ is also a subgroup of $\mathbf{R}$ such that no translation-invariant extension of $\lambda$ can make $H$ be measurable. Besides, the relation $\operatorname{card}(\mathbf{R} / H)=\operatorname{card}(\mathbf{Q})=\omega$ holds true, where $\omega$ denotes the least infinite cardinal (which may be identified with the set of all natural numbers).

There are some other constructions which establish that there exist subgroups $F$ of $\mathbf{R}$ nonmeasurable with respect to $\lambda$, but satisfying the inequality $\operatorname{card}(\mathbf{R} / F)>\omega$. For instance, by applying the method of transfinite recursion, it is not difficult to construct a subgroup $F$ of $\mathbf{R}$ satisfying the above-mentioned inequality and the relation $\lambda_{*}(\mathbf{R} \backslash F)=0$, where $\lambda_{*}$ stands for the inner measure associated with $\lambda$. The construction of such an $F$ can be done similarly to the classical Bernstein construction (cf. [14], [16], [17]). The advantage of $F$ over $H$ is that $F$ can be made measurable with respect to an appropriate translationinvariant extension of $\lambda$. This fact is not accidental. It remains valid for any uncountable commutative group (see Lemma 2 below). We are going to give an application of one purely algebraic statement to the question of the existence of nonmeasurable subgroups and to the problem of extending nonzero $\sigma$-finite invariant (quasiinvariant) measures.

In what follows the symbol $(G, \cdot)$ will denote an arbitrary group and $(G,+)$ will denote an arbitrary commutative group. We do not suppose that $G$ is equipped with a topology compatible with its algebraic structure (equivalently, we may assume that $G$ is endowed with its discrete topology). Let $\mu$ be a measure defined on some $G$-invariant $\sigma$-algebra of subsets of $(G,+)$. We recall that $\mu$ is invariant under $G$ (or, in short, is $G$-invariant) if, for any set $X \in$ $\operatorname{dom}(\mu)$ and for any element $g \in G$, we have $\mu(g+X)=\mu(X)$. We also recall that $\mu$ is quasiinvariant under $G$ (or, in short, is $G$-quasiinvariant) if, for any 
$\mu$-measure zero set $X$ and for any $g \in G$, we have $\mu(g+X)=0$. Clearly, every $G$-invariant measure is $G$-quasiinvariant. The converse assertion is not true, in general.

Let $H \subset \mathbf{R}$ be as above. We have already mentioned that $H$ is absolutely nonmeasurable with respect to the class of all translation-invariant extensions of $\lambda$. On the other hand, one can demonstrate that $H$ becomes measurable with respect to an appropriate quasiinvariant extension of $\lambda$. The following statement contains a much stronger result which shows that there are many possibilities for such quasiinvariant extensions of $\lambda$.

Theorem 1. There exist continuumly many measures $\nu$ on $\mathbf{R}$, which extend $\lambda$, are quasiinvariant under the group of all translations of $\mathbf{R}$ and satisfy the relation $H \in \operatorname{dom}(\nu)$.

Proof. We begin by observing that $H$ is $\lambda$-thick in $\mathbf{R}$, i.e., $\lambda_{*}(\mathbf{R} \backslash H)=0$. Further, we introduce the family of sets

$$
\left\{H_{k}: k<\omega\right\}=\{q+H: q \in \mathbf{Q}\} .
$$

Obviously, for any $h \in \mathbf{R}$, the family $\left\{h+H_{k}: k<\omega\right\}$ coincides with $\left\{H_{\phi(k)}\right.$ : $k<\omega\}$ where $\phi$ is some permutation of $\omega$. We thus claim that $\left\{H_{k}: k<\omega\right\}$ is a countable translation-invariant partition of $\mathbf{R}$ into $\lambda$-thick sets. Now, define the class of sets

$$
\mathcal{S}=\left\{\cup_{k<\omega}\left(H_{k} \cap X_{k}\right): X_{k} \in \operatorname{dom}(\lambda) \text { for all } k<\omega\right\} .
$$

It can easily be checked that $\mathcal{S}$ is a translation-invariant $\sigma$-algebra of subsets of R. Fix a sequence $\left\{a_{k}: k<\omega\right\}$ of strictly positive reals such that $\sum_{k<\omega} a_{k}=1$. Take an arbitrary element $\cup_{k<\omega}\left(H_{k} \cap X_{k}\right)$ from $\mathcal{S}$ and put

$$
\nu\left(\cup_{k<\omega}\left(H_{k} \cap X_{k}\right)\right)=\sum_{k<\omega} a_{k} \lambda\left(X_{k}\right) .
$$

In this way we define a certain functional $\nu$ on $\mathcal{S}$. Indeed, the definition of $\nu$ is correct in view of the thickness of all $H_{k}$. For the same reason, the functional $\nu$ is countably additive, so $\nu$ is a measure on $\mathcal{S}$. If $X \in \operatorname{dom}(\lambda)$, then

$$
\nu(X)=\nu\left(\cup_{k<\omega}\left(H_{k} \cap X\right)\right)=\sum_{k<\omega} a_{k} \lambda(X)=\lambda(X),
$$

which shows that $\nu$ extends $\lambda$. Finally, if we have

$$
\nu\left(\cup_{k<\omega}\left(H_{k} \cap X_{k}\right)\right)=\sum_{k<\omega} a_{k} \lambda\left(X_{k}\right)=0,
$$

then, taking into account the inequalities $a_{k}>0$ for all $k<\omega$, we get

$$
\lambda\left(X_{k}\right)=0(k<\omega)
$$

which, for any $h \in \mathbf{R}$, implies that

$$
\nu\left(h+\cup_{k<\omega}\left(H_{k} \cap X_{k}\right)\right)=\nu\left(\cup_{k<\omega}\left(H_{\phi(k)} \cap\left(h+X_{k}\right)\right)\right)=\sum_{k<\omega} a_{\phi(k)} \lambda\left(h+X_{k}\right)=0 .
$$


Therefore, $\nu$ is quasiinvariant under the group of all translations of $\mathbf{R}$ (a similar argument yields that $\nu$ is also quasiinvariant under the symmetry of $\mathbf{R}$ ). Since $\nu$ depends on the choice of a sequence of strictly positive reals $\left\{a_{k}: k<\omega\right\}$, we conclude that there are at least continuumly many quasiinvariant extensions of $\lambda$ for which the Vitali set $H$ becomes measurable. Theorem 1 has thus been proved.

Let $(G, \cdot)$ be an uncountable group. If $\mu$ is a nonzero $\sigma$-finite left $G$-quasiinvariant measure on $G$, then there exists a subset of $G$ nonmeasurable with respect to $\mu$ (see [3], [8]). This result is based on the classical theorem of Ulam stating that the first uncountable cardinal $\omega_{1}$ is not real-valued measurable (see [16], [17], [24]). For an uncountable commutative group $(G,+)$ equipped with a nonzero $\sigma$-finite $G$-quasiinvariant measure $\mu$, it can be shown that there exists a subgroup of $(G,+)$ nonmeasurable with respect to $\mu$ (in general, the same result does not hold for an uncountable noncommutative group equipped with a nonzero $\sigma$-finite left invariant measure). Moreover, there are uncountably many subgroups of $(G,+)$ nonmeasurable with respect to $\mu$ (see, e.g., Ch. 13 of [11]). Here we are interested in some results closely connected with the existence of those nonmeasurable subgroups of $(G,+)$ which can be used for obtaining extensions of invariant (quasiinvariant) measures given on $G$.

The algebraic structure of an arbitrary commutative group is investigated more or less thoroughly. The following statement yields a description of this structure: any commutative group $(G,+)$ admits a representation $G=\cup\left\{G_{n}\right.$ : $n<\omega\}$ where $\left\{G_{n}: n<\omega\right\}$ is an increasing (by inclusion) sequence of subgroups of $G$ and, for each $n<\omega$, the group $G_{n}$ is a direct sum of cyclic groups (finite or infinite).

For the proof of this statement, see, e.g., [15]. Suppose now that our $(G,+)$ is uncountable. Then we may assume, without loss of generality, that all $G_{n}$ in the representation above are also uncountable. Let us express $G_{n}$ in the form of a direct sum

$$
G_{n}=\sum_{i \in I_{n}} G_{i}
$$

where all $G_{i}$ are cyclic groups (finite or infinite) and card $\left(G_{i}\right) \geq 2$. According to our assumption, we have $\operatorname{card}\left(I_{n}\right)>\omega$. Further, let us represent $I_{n}$ in the form

$$
I_{n}=\cup\left\{I_{n, k}: k<\omega\right\}
$$

where $\left\{I_{n, k}: k<\omega\right\}$ is a partition of $I_{n}$ and $\operatorname{card}\left(I_{n, k}\right)>\omega$ for each $k<\omega$. Finally, let us put

$$
G_{n, k}=\sum_{i \in I_{n, 0} \cup I_{n, 1} \cup \cdots \cup I_{n, k}} G_{i}
$$

and consider the countable family $\left\{G_{n, k}: n<\omega, k<\omega\right\}$ of subgroups of $G$. It is not difficult to verify the following relations:

(1) $\operatorname{card}\left(G_{n} / G_{n, k}\right)>\omega$ for all $n<\omega$ and $k<\omega$;

(2) $\cup\left\{G_{n, k}: k<\omega\right\}=G_{n}$ for all $n<\omega$.

Relation (1) implies that $\operatorname{card}\left(G / G_{n, k}\right)>\omega$ for all $n<\omega$ and $k<\omega$. 
Relation (2) implies at once that $G=\cup\left\{G_{n, k}: n<\omega, k<\omega\right\}$.

We thus obtain the following fact:

Lemma 1. Any uncountable commutative group $(G,+)$ admits a representation $G=\cup\left\{G_{j}: j \in J\right\}$ where $J$ is a countable set, $G_{j}$ is a subgroup of $G$ for each $j \in J$, and $\operatorname{card}\left(G / G_{j}\right)>\omega$.

This fact enables us to give a solution of the Sierpiński problem ([23], Problem 1) on extensions of invariant measures for uncountable commutative groups by using the existence of certain nonmeasurable subgroups. For this purpose, we need the next simple auxiliary proposition.

Lemma 2. Let $(G,+)$ be an uncountable commutative group, $\mu$ be a $\sigma$-finite $G$-invariant ( $G$-quasiinvariant) measure on $G$ and let $G_{0}$ be a subgroup of $G$ such that $\operatorname{card}\left(G / G_{0}\right)>\omega$. Then there exists a $G$-invariant ( $G$-quasiinvariant) measure $\mu^{\prime}$ on $G$ extending $\mu$ and satisfying the equality $\mu^{\prime}\left(G_{0}\right)=0$.

Proof. Let us introduce the family of sets in $G$ :

$\mathcal{I}=\left\{X: X\right.$ can be covered by countably many translates of $\left.G_{0}\right\}$.

It is easy to see that $\mathcal{I}$ is a translation-invariant $\sigma$-ideal of sets in $G$. Moreover, if $X \in \mathcal{I}$, then for some countable family $\left\{g_{i}: i \in I\right\} \subset G$ we have

$$
X \subset \cup\left\{g_{i}+G_{0}: i \in I\right\} .
$$

Therefore $X \cap(h+X)=\varnothing$ for each element $h \in G$ not belonging to the set $\cup\left\{g_{i}-g_{j}+G_{0}: i \in I, j \in I\right\}$. This implies (by easy transfinite induction) that there exists an uncountable family $\left\{h_{\xi}: \xi<\omega_{1}\right\}$ of elements of $G$ such that the family $\left\{h_{\xi}+X: \xi<\omega_{1}\right\}$ consists of pairwise disjoint sets. We thus conclude that $\mu_{*}(X)=0$ (in view of the $\sigma$-finiteness and $G$-quasiinvariance of $\mu$ ).

Now, we can apply the standard method of extending a given measure $\mu$ (see, e.g., [22], [23]). Namely, we suppose (without loss of generality) that $\mu$ is complete and put

$$
\mathcal{S}^{\prime}=\{Y \triangle X: Y \in \operatorname{dom}(\mu), X \in \mathcal{I}\}
$$

Further, we define

$$
\mu^{\prime}(Y \triangle X)=\mu(Y)
$$

for any set $Y \triangle X \in \mathcal{S}^{\prime}$. An easy verification shows that $\mu^{\prime}$ is well defined and is a complete $G$-invariant ( $G$-quasiinvariant) measure extending $\mu$. According to this definition, $\mu^{\prime}(X)=0$ for all members $X$ of the $\sigma$-ideal $\mathcal{I}$. In particular, we have $\mu^{\prime}\left(G_{0}\right)=0$. This ends the proof of the lemma.

From the above lemmas we readily obtain the following result.

Theorem 2. Let $(G,+)$ be an uncountable commutative group. There exists a countable family $\left\{G_{j}: j \in J\right\}$ of subgroups of $G$ such that:

1) for any nonzero $\sigma$-finite $G$-invariant ( $G$-quasiinvariant) measure $\mu$ on $G$, at least one subgroup $G_{j}$ is nonmeasurable with respect to $\mu$; 
2) if $G_{j}$ is nonmeasurable with respect to $\mu$, then there exists a $G$-invariant $\left(G\right.$-quasiinvariant) measure $\mu^{\prime}$ on $G$ extending $\mu$ and satisfying the relation $\mu^{\prime}\left(G_{j}\right)=0$.

Proof. Let $\left\{G_{j}: j \in J\right\}$ be as in Lemma 1, and let $\mu$ be an arbitrary nonzero $\sigma$-finite $G$-invariant ( $G$-quasiinvariant) measure on $G$. By virtue of the equality $G=\cup\left\{G_{j}: j \in J\right\}$, we may write

$$
0<\mu(G) \leq \sum_{j \in J} \mu^{*}\left(G_{j}\right)
$$

where $\mu^{*}$ denotes the outer measure associated with $\mu$. Consequently, there exists $G_{j}$ such that $\mu^{*}\left(G_{j}\right)>0$. In view of the relation $\operatorname{card}\left(G / G_{j}\right)>\omega$, we easily infer that $G_{j}$ is nonmeasurable with respect to $\mu$. Finally, applying Lemma 2, we conclude that, for the same $G_{j}$, there exists a $G$-invariant ( $G$-quasiinvariant) measure $\mu^{\prime}$ on $G$ extending $\mu$ and satisfying the equality $\mu^{\prime}\left(G_{j}\right)=0$. This completes the proof.

By using a more complicated argument, Theorem 2 can be extended to the class of all uncountable solvable groups. Recall that a group $(G, \cdot)$ is solvable if there exists a finite sequence $\left(\Gamma_{0}, \Gamma_{1}, \ldots, \Gamma_{n}\right)$ of subgroups of $G$ such that:

(a) $\Gamma_{0}=G$ and $\operatorname{card}\left(\Gamma_{n}\right)=1$;

(b) for each integer $k \in[0, n-1]$, the group $\Gamma_{k+1}$ is normal in $\Gamma_{k}$ and the quotient group $\Gamma_{k} / \Gamma_{k+1}$ is commutative.

We say that a set $X \subset G$ is $G$-absolutely negligible in $G$ if, for any nonzero $\sigma$-finite left $G$-invariant (left $G$-quasiinvariant) measure $\mu$ on $G$, there exists a left $G$-invariant (left $G$-quasiinvariant) measure $\mu^{\prime}$ on $G$ extending $\mu$ and such that $\mu^{\prime}(X)=0$. Some properties of $G$-absolutely negligible sets are considered in [10]. For instance, it is proved there that the following two assertions are equivalent:

(c) $X$ is $G$-absolutely negligible in $G$;

(d) for any countable family $\left\{g_{i}: i \in I\right\} \subset G$, there exists a countable family $\left\{h_{j}: j \in J\right\} \subset G$ such that $\cap_{j \in J} h_{j}\left(\cup_{i \in I} g_{i} X\right)=\varnothing$.

Remark 1. In a certain sense, absolutely negligible sets may be regarded as small subsets of a given uncountable group. However, the group operation applied to such subsets sometimes yields a nonsmall set. More precisely, it can be demonstrated that, for every uncountable solvable group $(G, \cdot)$, there exist two $G$-absolutely negligible sets $A$ and $B$ satisfying the equality $A \cdot B=G$.

The next lemma follows from the equivalence of assertions (c) and (d) (cf. also $[27])$.

Lemma 3. Let $(G, \cdot)$ be a group, $H$ be a normal subgroup of $G$ such that $\operatorname{card}(G / H) \leq \omega$, and let $X$ be an $H$-absolutely negligible subset of $H$. Then $X$ is $G$-absolutely negligible in $G$.

We say that a family $\left\{G_{j}: j \in J\right\}$ of subgroups of $G$ is admissible if:

(i) $\operatorname{card}(J) \leq \omega$; 
(ii) there exists a countable family $\left\{g_{k}: k \in K\right\}$ of elements of $G$ such that $G=\cup_{k \in K} g_{k}\left(\cup\left\{G_{j}: j \in J\right\}\right)$

(iii) each group $G_{j}(j \in J)$ is $G$-absolutely negligible in $G$.

It can easily be seen that if $\left\{G_{j}: j \in J\right\}$ is an admissible family for $G$ and $\mu$ is an arbitrary nonzero $\sigma$-finite left $G$-invariant (left $G$-quasiinvariant) measure on $G$, then at least one group $G_{j}$ is nonmeasurable with respect to $\mu$. Therefore, $\mu$ can be extended to a left $G$-invariant (left $G$-quasiinvariant) measure $\mu^{\prime}$ on $G$ such that $\mu^{\prime}\left(G_{j}\right)=0$ (cf. Lemma 2).

Lemma 4. For every uncountable solvable group $(G, \cdot)$, there exists an admissible family of subgroups.

Proof. Let $\left(\Gamma_{0}, \Gamma_{1}, \ldots, \Gamma_{n}\right)$ be a finite family of subgroups of $G$ satisfying (a) and (b). We use induction on $n$. Let $\phi: \Gamma_{0} \rightarrow \Gamma_{0} / \Gamma_{1}$ denote the canonical epimorphism. Only two cases are possible.

1. $\operatorname{card}\left(\Gamma_{0} / \Gamma_{1}\right) \leq \omega$. In this case, we obviously have $\operatorname{card}\left(\Gamma_{1}\right)>\omega$. By inductive assumption, there exists an admissible family $\left\{G_{j}: j \in J\right\}$ of subgroups of $\Gamma_{1}$. Taking into account Lemma 3 and the inequality card $\left(\Gamma_{0} / \Gamma_{1}\right) \leq \omega$, we claim that the same family $\left\{G_{j}: j \in J\right\}$ is admissible for $\Gamma_{0}=G$.

2. $\operatorname{card}\left(\Gamma_{0} / \Gamma_{1}\right)>\omega$. In view of Lemma 1 , there exists an admissible family $\left\{G_{j}: j \in J\right\}$ of subgroups of the commutative group $\Gamma_{0} / \Gamma_{1}$. Now, it is not difficult to verify that the family of groups $\left\{\phi^{-1}\left(G_{j}\right): j \in J\right\}$ is admissible for the original group $\Gamma_{0}=G$.

This completes the proof of Lemma 4.

The two preceding lemmas immediately imply an analog of Theorem 2 for any uncountable solvable group $(G, \cdot)$, in terms of nonzero $\sigma$-finite left invariant (left quasiinvariant) measures on $G$. Namely, we have the next statement.

Theorem 3. Let $(G, \cdot)$ be an uncountable solvable group. There exists a family $\left\{G_{j}: j \in J\right\}$ of subgroups of $G$ satisfying the following relations:

1) $\left\{G_{j}: j \in J\right\}$ is admissible;

2) for any nonzero $\sigma$-finite left $G$-invariant (left $G$-quasiinvariant) measure $\mu$ on $G$, at least one subgroup $G_{j}$ is nonmeasurable with respect to $\mu$;

3) if $G_{j}$ is nonmeasurable with respect to $\mu$, then there exists a left $G$-invariant (left $G$-quasiinvariant) measure $\mu^{\prime}$ on $G$ extending $\mu$ and such that $\mu^{\prime}\left(G_{j}\right)=0$.

Remark 2. Let $(G, \cdot)$ be a connected locally compact topological group and let $D$ be a subgroup of $G$. Denote by $\nu$ the completion of the left Haar measure on $G$. Then we have either $\nu(D)=0$ or $\nu_{*}(G \backslash D)=0$. In the second case, the group $D$ is called thick with respect to $\nu$. Obviously, a thick subgroup is everywhere dense in the original group $G$. Moreover, if a thick subgroup $D$ is proper (i.e., $G \neq D$ ), then $D$ is nonmeasurable with respect to $\nu$. Indeed, supposing that $D$ is $\nu$-measurable (hence is of full measure) and taking any element $g \in G \backslash D$, we get $g D \cap D=\varnothing$, which is impossible in view of the relation

$$
\nu(g D)=\nu(D)>0
$$


Note that there exists an uncountable commutative $\sigma$-compact locally compact connected topological group $(G,+)$, which does not admit proper everywhere dense subgroups (see [20]). This fact shows that, in general, we cannot apply the Bernstein-type construction in order to obtain a nonmeasurable (with respect to the Haar measure) subgroup of $G$. On the other hand, the existence of such a subgroup follows directly from Theorem 2 and it should be underlined that no topological technique is needed for establishing this result. It should also be mentioned that every uncountable $\sigma$-compact locally compact group $G$ is a resolvable topological space, i.e., $G=A \cup B$, where $A$ and $B$ are two disjoint everywhere dense subsets of $G$. Indeed, a well-known theorem of Hewitt states that every locally compact topological space without isolated points is resolvable.

In various situations a topological structure of a given topological group does not admit any nonzero $\sigma$-finite quasiinvariant measure. For instance, it can be proved that if $G$ is a proper uncountable analytic (or co-analytic) subgroup of $\mathbf{R}$, then there exists no nonzero $\sigma$-finite $G$-quasiinvariant Borel measure on $G$ (see [9]). Similarly, if $E$ is an arbitrary infinite-dimensional Banach space, then there does not exist a nonzero $\sigma$-finite $E$-quasiinvariant Borel measure on $E$ (see, e.g., [21] where the case of an infinite-dimensional Hilbert space $E$ is considered in detail). Taking into account examples of this kind, it makes sense to ignore any topological concepts and to pose the following general question:

Let $(G, \cdot)$ be an uncountable solvable group. Does there exist a nonzero $\sigma$ finite measure defined on sufficiently large class of subsets of $G$ and invariant under all left translations?

Theorem 3 shows that any such measure $\mu$ is defined on a proper subclass of the family of all subsets of $G$ and that $\mu$ can be strictly extended by using some $\mu$-nonmeasurable subgroup of $G$. However, the method of extending measures described in Theorems 2 and 3 does not essentially change the class of measurable sets. For instance, if the original measure $\mu$ on $G$ is separable, then the extended measure $\mu^{\prime}$ remains separable, because all $\mu^{\prime}$-measurable sets are obtained with the aid of members of a certain $G$-invariant $\sigma$-ideal in $G$ which does not change the metrical structure of $\mu$. Therefore, it is reasonable to ask whether $G$ admits a nonseparable $\sigma$-finite left $G$-invariant measure. It turns out that the answer to the latter question is positive under an additional set-theoretic assumption.

Theorem 4. Let $(G, \cdot)$ be an uncountable solvable group. Assuming the Continuum Hypothesis, there exists a nonatomic nonseparable $\sigma$-finite left $G$ invariant measure on $G$.

For the proof, see [12]. An essential role in that proof is played by the following algebraic fact: if $(A,+)$ is an uncountable commutative group, then there exists a homomorphic image $(B,+)$ of $(A,+)$ such that $\operatorname{card}(B)=\omega_{1}$. Evidently, this fact fails to be true for uncountable non-commutative groups.

We do not know whether $\mathbf{C H}$ is necessary for the validity of the conclusion of Theorem 4. 
Also, it is unknown whether any uncountable group $(G, \cdot)$ admits a nonatomic nonseparable $\sigma$-finite left invariant measure. This is so if $\operatorname{card}(G) \geq 2^{\omega}$ and, simultaneously, $\operatorname{card}(G)$ is not cofinal with $\omega$. However, if these conditions do not hold for $G$, the question remains open. Note that if $(G, \cdot)$ is an uncountable $\sigma$-compact locally compact topological group, then $\operatorname{card}(G)=(\operatorname{card}(G))^{\omega}($ see, e.g., [2]); therefore, in this case the above-mentioned conditions are satisfied automatically.

Concluding this paper, let us recall that the first two (essentially different) constructions of nonseparable invariant extensions of the Lebesgue measure were carried out by Kakutani and Oxtoby [7], and Kodaira and Kakutani [13], respectively. Their constructions admit a straightforward generalization to a certain class of locally compact groups (cf. [4], Chapter 4). More information concerning extensions of measures invariant under various transformation groups can be found in [1], [5], [6], [18], [19], [22], [25], [26], [28].

\section{REFERENCES}

1. K. Ciesielski, Algebraically invariant extensions of $\sigma$-finite measures on Euclidean space. Trans. Amer. Math. Soc. 318(1990), No. 1, 261-273.

2. W. W. Comfort, Topological groups. Handbook of set-theoretic topology, 1143-1263, North-Holland, Amsterdam, 1984.

3. P. Erdös and R. D. Mauldin, The nonexistence of certain invariant measures. Proc. Amer. Math. Soc. 59 1976), No. 2, 321-322.

4. E. HewitT and K. A. Ross, Abstract harmonic analysis. Vol. I: Structure of topological groups. Integration theory, group representations. Die Grundlehren der mathematischen Wissenschaften, Bd. 115. Academic Press, Inc., Publishers, New York; Springer-Verlag, Berlin-Göttingen-Heidelberg, 1963.

5. A. Hulanicki, Invariant extensions of the Lebesgue measure. Fund. Math. 51 (1962/1963), 111-115.

6. A. Hulanicki and C. Ryll-Nardzewski, Invariant extensions of the Haar measure. Colloq. Math. 42(1979), 223-227.

7. S. Kakutani and J. C. Oxtoby, Construction of a non-separable invariant extension of the Lebesgue measure space. Ann. of Math. (2) 52(1950), 580-590.

8. A. B. Kharazishvili, Certain types of invariant measures. (Russian) Dokl. Akad. Nauk SSSR 222(1975), No. 3, 538-540.

9. A. B. Kharazishvili, Some remarks on quasiinvariant and invariant measures. Real Anal. Exchange 24(1998/99), No. 1, 427-434.

10. A. B. Kharazishvili, Transformation groups and invariant measures. Set-theoretical aspects. World Scientific Publishing Co., Inc., River Edge, NJ, 1998.

11. A. B. Kharazishvili, Nonmeasurable sets and functions. North-Holland Mathematics Studies, 195. Elsevier Science B.V., Amsterdam, 2004.

12. A. Kharazishvili and A. Kirtadze, Nonseparable left-invariant measures on uncountable solvable groups. Proc. A. Razmadze Math. Inst. 139(2005), 45-52.

13. K. Kodaira and S. Kakutani, A non-separable translation invariant extension of the Lebesgue measure space. Ann. of Math. (2) 52(1950), 574-579.

14. K. Kuratowski, Topology. Vol. I. New edition, revised and augmented. (Translated from the French) Academic Press, New York-London; Państwowe Wydawnictwo Naukowe, Warsaw, 1966. 
15. A. G. Kurosh, Theory of groups. (Russian) Nauka, Moscow, 1967.

16. J. C. Morgan, II, Point set theory. Monographs and Textbooks in Pure and Applied Mathematics, 131. Marcel Dekker, Inc., New York, 1990.

17. J. C. Охтову, Measure and category. A survey of the analogies between topological and measure spaces. Graduate Texts in Mathematics, Vol. 2. Springer-Verlag, New YorkBerlin, 1971.

18. A. PelC, Invariant measures and ideals on discrete groups. Dissertationes Math. (Rozprawy Mat.) 255(1986), 47 pp.

19. Sh. S. Pkhakadze, The theory of Lebesgue measure. (Russian) Trudy Tbiliss. Mat. Inst. Razmadze 25(1958), 3-271.

20. M. Rajagopalan and H. Subrahmanian, Dense subgroups of locally compact groups. Colloq. Math. 35(1976), No. 2, 289-292.

21. A. V. Skorokhod, Integration in Hilbert space. (Translated from the Russian) Ergebnisse der Mathematik und ihrer Grenzgebiete, Band 79. Springer-Verlag, New YorkHeidelberg, 1974.

22. E. Szpilrajn (E. Marczewski), Sur l'extension de la mesure lebesguienne. Fund. Math. 25(1935), 551-558.

23. E. Szpilrajn (E. Marczewski), On problems of the theory of measure. (Russian) Uspekhi Mat. Nauk 1(1946), No. 2 (12), 1946, 179-188.

24. S. Ulam, Zur Masstheorie in der allgemeinen Mengenlehre. Fund. Math. 16(1930), 140150.

25. P. ZAKRzEWSKI, Extensions of isometrically invariant measures on Euclidean spaces. Proc. Amer. Math. Soc. 110 (1990), No. 2, 325-331.

26. P. ZAKRZEWSKi, Extending invariant measures on topological groups. Papers on general topology and applications (Amsterdam, 1994), 218-222, Ann. New York Acad. Sci., 788, New York Acad. Sci., New York, 1996.

27. A. Kharazishvili, On absolutely negligible sets in uncountable solvable groups. Georgian Math. J. 12(2005), No. 2, 255-260.

28. G. Pantsulaia, An application of independent families of sets to the measure extension problem. Georgian Math. J. 11(2004), No. 2, 379-390.

(Received 17.02.2007)

Authors' addresses:

A. Kharazishvili

A. Razmadze Mathematical Institute

1, M. Aleksidze St., Tbilisi 0193, Georgia

E-mail: kharaz2@yahoo.com

A. Kirtadze

I. Vekua Institute of Applied Mathematics

I. Javakhishvili Tbilisi State University

2, University St., Tbilisi 0143

Georgia

E-mail: kirtadze2@yahoo.com 